\title{
Tendencias en la investigación de alumnos de posgrado en ELE fundamentada en necesidades y competencias docentes
}

\author{
María Pilar López-García. Universidad de Granada \\ Jerónimo Morales-Cabezas. Universidad de Granada - ORCID 0000-0002-3446-620X
}

Recepción: 29/06/2018 | Aceptado: 17/04/2019

Correspondencia a través de ORCID: María Pilar López-García

0000-0002-9884-6332

Citar: López, P y Morales, J (2019). Tendencias en la investigación de alumnos de posgrado en ELE fundamentada en necesidades y competencias docentes. ReiDoCrea - Monográfico sobre Perspectivas transnacionales en la enseñanza de lenguas, 8(3), 158-169.

\begin{abstract}
Resumen: Los gestores académicos debemos hacer continuas reflexiones sobre las necesidades de los alumnos en formación para diseñar programas didácticos acordes a las nuevas tendencias en la enseñanza de lenguas. Los futuros profesores de español como lengua extranjera (ELE) y/o segunda lengua (L2) que están en proceso de formación se crean -como cualquier individuo que se desenvuelve en un ámbito formativo- diversas expectativas personales cuando acceden al posgrado específico. En esta investigación partimos de un estudio previo sobre necesidades formativas de los futuros docentes de ELE -alumnos de posgrado- y la posterior investigación desarrollada al finalizar un máster. Los principales objetivos de este trabajo consisten, en primer lugar, en analizar un conjunto de necesidades expresadas por este tipo de alumnado y relacionarlas con las competencias docentes descritas en este amplio ámbito. En segundo lugar, estudiaremos una muestra de trabajos fin de máster (TFM) derivados de las investigaciones hechas por los alumnos. Nuestro propósito es ver qué tipo de tendencias o líneas de investigación son predominantes en los diferentes cursos académicos y si se adecuan o no al perfil competencial de ELE, para distinguir qué actividad formativa en este ámbito es más adecuada en función de las necesidades profesionales de estos futuros docentes.
\end{abstract}

Palabras clave: Competencias de docente, Lengua extranjera.

Trends in the research of postgraduate students in SFL based on teacher needs and competences

Abstract: Academic managers must constantly reflect on the needs of learners in training in order to design teaching programs in line with the new trends in language teaching. Future teachers of Spanish as a Foreign Language (SFL) and/or second language (L2) that are in the process of formation are created -as any individual that is carried out in a formative field- different personal expectations when they accede to the specific postgraduate degree. In this research we start from a previous study on the training needs of the future teachers of SFL - postgraduate students- and the subsequent research developed at the end of the master. The main objectives of this work are, first of all, to analyze a set of needs expressed by this type of student and to relate them to the teaching competences described in this broad field. Secondly, we will look at a sample of master's dissertation derived from the research done by the students. Our purpose is to see what kind of trends or lines of research are predominant in the different academic courses and whether or not they fit the competency profile of SFL, to distinguish which training activity in this field is best suited to the professional needs of these future teachers.

Keywords: Teacher qualifications, Foreign languages.

\section{Introducción}

La formación del profesorado de español como lengua extranjera (ELE) y/o segunda lengua (E/L2) en másteres oficiales y en títulos propios se ha sustentado en un amplio conjunto de modelos competenciales en los que se aglutinan el conocimiento teórico de la acción docente (formación lingüística-gramatical, gestión del proceso de enseñanzaaprendizaje, por citar dos factores destacados) y la formación práctica (aplicación del conocimiento teórico y planificación del proceso de enseñanza). Prueba de ello es la prolija publicación de trabajos de investigación en las dos últimas décadas (Perrenoud, 2001; Battaner Arias, 2002, Verdía, 2010, 2013 y 2016; Níkleva y Rico-Martín, 2017; 
López-García, 2017) sobre competencias, saberes y acciones de este tipo de profesorado.

En estas investigaciones se apunta hacia diversos fundamentos en la formación como la actuación del docente en el aula $-y$ fuera de ella-, el desarrollo de habilidades socioprofesionales (trabajo en equipo en una determinada institución académica) y la extensión de una formación continua a lo largo de su vida profesional.

De otro lado, la formación de profesores necesita reflexiones continuas sobre la evolución y el desarrollo de los programas de formación y, de otro, es imprescindible el diagnóstico de necesidades de los futuros profesionales desde los inicios de su etapa formativa con objeto de mejorar la planificación de la formación y la calidad de las enseñanzas del posgrado en este ámbito formativo.

Los resultados finales del periodo formativo se aprecian en un trabajo se investigación que culmina con la realización de un trabajo fin de máster (TFM), el cual se inserta en una línea de investigación concreta sobre una temática explícita que muestra el rendimiento de los conocimientos adquiridos y su ulterior aplicación. Estas producciones suelen ser tan amplias como variadas; por esa razón, en 2013 comenzamos a recopilar datos sobre las producciones de trabajos en dos másteres, uno de título oficial impartido en línea (Máster en Lingüística Aplicada a la Enseñanza del Español como Lengua Extrajera de la Universidad de Jaén) desde el curso académico 2008-2009 hasta el curso 2016-2017, y otro de título propio impartido presencialmente desde el curso académico 2004-2005 hasta el curso 2015-2016 (Máster Propio para la Formación del Profesorado de Español como Lengua Extranjera de la Universidad de Granada). La extinción de ambos másteres nos llevó a recabar y cruzar datos sobre dichas producciones, aunque aquí vamos a centrarnos en los resultados de la investigación obtenidos en el Título Propio, en el que hemos sido gestores, docentes y tutores de TFM. Con ello pretendemos, en primer lugar, profundizar en el estudio de las necesidades de formación de los futuros profesores, en segundo lugar, comprobar si estas necesidades se ajustan a los perfiles competenciales descritos del profesorado de ELE y E/L2 y, en tercer lugar, analizar la temática de los productos finales con objeto de comprobar cuáles son las líneas de investigación predominantes y los temas recurrentes.

\section{El diagnóstico de necesidades en la formación del profesorado}

De todos es sabido que los cambios en el paradigma de los profesionales de la enseñanza en general han evolucionado a pasos agigantados en el siglo XXI. Se ha pasado de una noción tradicional basada en la transmisión de conocimientos por parte del docente -siendo el profesor un elemento único activo y el discente un elemento receptivo de dichos conocimientos- a una concepción del proceso de enseñanzaaprendizaje fundamentado en saberes, competencias y acciones en las que el alumno es un sujeto que participa activamente en el proceso de aprendizaje y es responsable del mismo.

Actualmente, los diseños de programas académicos tienen en cuenta el análisis de necesidades para mejorar la calidad de la docencia. Prueba de ello son los trabajos que han partido de este diagnóstico de necesidades formativas y docentes (García Llamas, 1998; Cano, 2005; Martín Peris, 2015; Níkleva y Rico-Martín, 2017; McMullin, 2016, entre otros).

Desde la perspectiva formativa y didáctica, la definición de necesidades ha procurado coordinar una serie de objetivos de aprendizaje con la finalidad de crear unos programas de formación sólidos y ajustados a las carencias, las demandas e intereses de los alumnos (López y Morales, 2015). Por tanto, la consideración del análisis de necesidades se ha convertido en un factor esencial en toda planificación curricular. Precisamente en el citado trabajo se incide en un estudio basado en cuestionarios de preguntas abiertas realizadas a 106 sujetos al inicio del máster. De ellos se obtuvieron las siguientes necesidades y expectativas -discriminamos las plenamente objetivas de 
las concreciones subjetivas- sustentadas en patrones de estrategias universales de aprendizaje vinculadas a la formación docente, las cuales categorizamos en esta tabla:

\begin{tabular}{|cl|}
\hline 1. & La obtención una buena formación práctica \\
\hline 2. & La formación como docente \\
\hline 3. & La profundización en las destrezas docentes \\
\hline 4. & El conocimiento de las competencias del profesor de ELE \\
\hline 5. & La recepción de una buena orientación y formación profesional \\
\hline 6. & El conocimiento de la metodología y didáctica de la lengua extranjera \\
\hline 7. & La determinación y seguimiento de itinerarios de investigación \\
\hline 8. & La adquisición de tipos significativos de aprendizaje \\
\hline 9. & El aprendizaje de la docencia en contextos escolares \\
\hline 10. & La profundización en las relaciones alumno-profesor, alumno-alumno. \\
\hline 11. & El desarrollo de estrategias de comunicación docente y profesional \\
\hline 12. & El aprendizaje de valores de transmisión cultural asociados a la lengua \\
\hline 13. & El fomento de la enseñanza-aprendizaje intercultural \\
\hline 14. & El conocimiento de los recursos docentes -TIC- para el desempeño profesional \\
\hline
\end{tabular}

Los resultados de este calado en las opiniones de los alumnos en formación de ELE nos condujeron a estos resultados en grandes líneas: interés en necesidades del ámbito de la docencia en general (puntos 2, 4, 6, 9, 11 y 14), 42.8\%, Sin embargo, los alumnos mostraron un menor interés por el ámbito de la investigación -solo un $7.1 \%$ - lo cual es indispensable en la posterior elaboración del TFM (trabajo fin de máster) para la obtención del título del máster.

\section{Necesidades formativas y competencias docentes}

En otro trabajo posterior (López-García, 2017) se afrontó la cuestión de las necesidades formativas del profesorado vinculándolas a los perfiles competenciales del profesor de ELE definidos como competencias clave por el Instituto Cervantes (2012:11) en las que se agrupan 8 competencias dentro de las cuales se incluyen 4 competencias específicas -32 en total-:

a) Organizar situaciones de aprendizaje: diagnosticar y atender las necesidades de los alumnos, promover el uso y la reflexión sobre la lengua, planificar secuencias didácticas y gestionar el aula.

b) Evaluar el aprendizaje y la actuación del alumno: servirse de herramientas y procedimientos de evaluación, garantizar buenas prácticas en la evaluación, promover una retroalimentación constructiva e implicar al alumno en la evaluación.

c) Implicar a los alumnos en el control de su propio aprendizaje: promover que el alumno gestione los recursos y medios disponibles para aprender, integrar en la enseñanza herramientas para reflexionar sobre el proceso de aprendizaje, promover que el alumno defina su propio proyecto de aprendizaje y motivar al alumno para que se responsabilice de su propio aprendizaje.

d) Facilitar la comunicación intercultural: implicarse en el desarrollo de la propia competencia intercultural, adaptarse a las culturas del entorno, fomentar el diálogo intercultural y promover que el alumno desarrolle su competencia intercultural.

e) Desarrollarse profesionalmente como profesor de la institución: analizar y reflexionar sobre la práctica docente, definir un plan personal de formación continua, implicarse en el desarrollo profesional del equipo docente y participar activamente en el desarrollo de la profesión.

f) Gestionar sentimientos y emociones en el desempeño de su trabajo: gestionar las propias emociones, motivarse en el trabajo, desarrollar las relaciones interpersonales e implicarse en el desarrollo de la inteligencia emocional del alumno. 
g) Participar activamente en la institución: trabajar en equipo en el centro, implicarse en los proyectos de mejora del centro, promover y difundir buenas prácticas en la institución y conocer la institución e integrarse en ella.

h) Servirse de las TIC para el desempeño de su trabajo: implicarse en el desarrollo de la propia competencia digital, desenvolverse en entornos digitales y con aplicaciones informáticas disponibles, aprovechar el potencial didáctico de las TIC y promover que el alumno se sirva de las TIC para su aprendizaje.

Para abordar el estudio se pasó otro cuestionario a 62 alumnos de posgrado al finalizar su periodo de formación. En él se pedía que expresaran cuáles eran, en su opinión, las tres competencias primordiales de un profesor de ELE. Las respuestas obtenidas se clasificaron en 7 categorías:

1. Dominio teórico de la acción docente: a) poseer formación una buena formación en contenidos pragmalingüísticos; b) conocer valores culturales e interculturales; c) dominar los aspectos metodológicos.

2. Formación práctica: a) saber aplicar y desarrollar los contenidos teóricos recibidos en su formación; b) realizar un gran número de horas prácticas; c) conocer todo tipo de materiales y recursos didácticos; d) saber planificar la enseñanza.

3. Dominio de la acción docente: a) saber diseñar actividades y tareas; b) hacer programaciones; c) planificar las clases; d) conocer los instrumentos de evaluación del aprendizaje.

4. Gestión del proceso de enseñanza-aprendizaje: a) considerar las necesidades de aprendizaje de los alumnos; b) crear situaciones propicias de aprendizaje; c) saber motivar a sus alumnos, c) desarrollar estrategias de comunicación con los alumnos.

5. Desarrollo de habilidades de interacción socioprofesional: a) saber trabajar en equipo;

b) saber mediar y resolver conflictos; c) implicarse en su centro de trabajo.

6. Formación continua: a) seguir cursos de formación, asistir a congresos y encuentros profesionales; b) estar atentos a los progresos metodológicos y tecnológicos.

7. Acción investigadora en su profesión: a) observar e investigar los procesos del aula;

b) emprender acciones innovadoras.

La valoración de estas categorías se puede observar en la siguiente tabla:

\begin{tabular}{|l|l|}
\hline 1. Dominio teórico de la acción docente & $15.1 \%$ \\
\hline 2. Formación práctica & $22.6 \%$ \\
\hline 3. Dominio de la acción docente & $19.4 \%$ \\
\hline 4. Gestión del proceso de enseñanza-aprendizaje & $17.7 \%$ \\
\hline 5. Desarrollo de habilidades de interacción socioprofesional & $11.3 \%$ \\
\hline 6. Formación continua & $8.1 \%$ \\
\hline 7. Acción investigadora en su profesión & $5.9 \%$ \\
\hline
\end{tabular}

Tabla 2. Categorías competenciales (López-García, 2017:81)

Los resultados obtenidos revelan que la categoría más valorada es la formación práctica (42 respuestas $=22.6 \%$ ), seguida del dominio de la acción docente (36 respuestas $=19.4 \%$ ) y la gestión del proceso de enseñanza-aprendizaje (33 respuestas $=17.4 \%$ ). El dominio teórico de la acción docente (28 respuestas $=15.1 \%$ ) y el desarrollo de habilidades de interacción socioprofesional (21 respuestas $=11.3 \%$ ) reciben porcentajes ligeramente más bajos. Las categorías referidas a la formación continua (15 respuestas $=8.1 \%$ ) y la acción investigadora en su profesión $(11=5.9 \%)$ son las menos apreciadas por los profesores noveles.

Si comparamos las creencias de los futuros profesores acerca del perfil competencial del profesor de ELE con las opiniones de los profesores experimentados -el documento Las competencias clave del profesorado de lenguas segundas y extranjeras, publicado 
en 2012 por el Instituto Cervantes se elaboró a partir de las opiniones de los profesores de la institución-, podemos observar que:

La categoría 1, dominio teórico de la acción docente coincide plenamente con la competencia clave a) del Instituto Cervantes (2012) titulada de la misma forma.

La categoría 2, formación práctica, a pesar de ser la categoría más valorada, solo coincide en un aspecto con la competencia a), organizar situaciones de aprendizaje, en la competencia específica "planificar secuencias didácticas".

La categoría 3, dominio de la acción docente, concuerda con la competencia a), organizar situaciones de aprendizaje, en la competencia específica sobre la gestión del aula y con la competencia b), evaluar el aprendizaje y la actuación del alumno, en la competencia específica "servirse de herramientas y procedimientos de evaluación".

La categoría 4, gestión del proceso de enseñanza-aprendizaje, coincide completamente con la competencia a), organizar situaciones de aprendizaje, en la competencia específica "diagnosticar y atender las necesidades de los alumnos", y "en promover el uso y la reflexión sobre la lengua" y con la competencia c) implicar a los alumnos en el control de su propio aprendizaje en la competencia específica "motivar al alumno para que se responsabilice de su propio aprendizaje".

La categoría 5, desarrollo de habilidades de interacción socioprofesional, se corresponde con la competencia g), participar activamente en la institución, en las competencias específicas "trabajar en equipo en el centro" y "conocer la institución e integrarse en ella".

La categoría 6 , formación continua, aun siendo una categoría poco valorada, concuerda con la competencia e), desarrollarse profesionalmente como profesor de la institución en la competencia específica "definir un plan personal de formación continua" y con la competencia clave h) servirse de las TIC para el desempeño de su trabajo, en la competencia específica "implicarse en el desarrollo de la propia competencia digital".

La categoría 7, aunque en un bajo porcentaje, es considerada por los alumnos que han terminado su etapa de formación e inician su periodo de investigación -realización del TFM-. Curiosamente el documento del Instituto Cervantes (2012) no la menciona y puede presuponer esta competencia entre sus docentes experimentados, pero se podría integrar una competencia clave más, la competencia investigadora, entre sus competencias clave, del mismo modo que ha sido descrita en otros elencos competenciales para los docentes de la educación superior (Mas Torelló, 2011; Saravia Gallardo, 2011).

\section{La competencia investigadora en los másteres}

Todos los másteres son cursos de especialización en una materia específica y están dirigidos a estudiantes de posgrado universitario. En ellos se pretende alcanzar una formación especializada y multidisciplinar dirigida a la consecución de logros académicos, de especialidades profesionales o de iniciación a la investigación.

Los estudios de máster exigen la evaluación continua del proceso de aprendizaje que se concreta finalmente en un trabajo de investigación -trabajo de fin de máster- cuya finalidad es acreditar las competencias adquiridas en el programa formativo; por tanto, el TFM es la prueba definitiva de la madurez y de la profesionalidad tras un periodo de formación en el que se vinculan saberes y competencias.

Por otro lado, la universidad del siglo XXI está centrada en la formación de discentes y en la cualificación de profesionales sustentada en los denominados estándares del conocimiento, lo cual lleva aparejados otros conceptos como la igualdad de oportunidades y la cohesión social. Por estas razones, la universidad de hoy demanda en el proceso de formación un conjunto capacidades articuladas entorno a conocimientos especializados para ser buenos profesionales y desarrollar diversas competencias. En concreto, y en el ámbito de los futuros docentes, no solo se trata de articular habilidades y actitudes capacitadoras, sino también de promover la acción 
investigadora y los procedimientos de innovación. Por ello, en los últimos años en el contexto universitario se ha venido hablando de la competencia investigadora (Más Torelló, 2011) o de la competencia científica, como ha apuntado Saravia Gallardo (2011). En definitiva, se trata de definir un conjunto integrado de conocimientos, habilidades, actitudes y valores para conseguir una enseñanza-aprendizaje de calidad científica.

Desde este punto de vista, Saravia Gallardo (2011:19-22) determina un "Marco Estructural de Competencias del Profesorado Universitario (MECPU)" basado en cuatro categorías en las que incluye la competencia científica y dentro de esta apunta estas tres dimensiones:

A) El saber del área de conocimiento (background teórico que supone el dominio de la disciplina).

B) La investigación integrada como motor del aprendizaje (conocimiento y aplicación sistemática de los criterios básicos del proceso de investigación).

C) La contribución a la generación y difusión de nuevo conocimiento científico (planificación y desarrollo de actividades de investigación científica, con la intención de profundizar en su aprendizaje, enriquecer su formación y aportar al progreso de la ciencia según el campo correspondiente).

En consecuencia, el hecho de incluir funciones investigadoras en el elenco competencial de la formación de futuros docentes nos ha llevado a hacer una revisión de los productos finales en los másteres que hemos estudiado. Con ello pretendemos analizar los resultados y ver hacia dónde se dirige la investigación en los trabajos de fin de máster y relacionarlo con las competencias presupuestas a los docentes, como veremos en los gráficos de los siguientes apartados.

\section{Tendencias en la investigación de los alumnos de posgrado}

El concepto investigación implica una multiplicidad de actividades para aquellos trabajos innovadores en los campos académico, profesional y tecnológico. A lo largo de la formación de posgrado, los estudiantes van adquiriendo una serie de competencias rentables para iniciar el TFM; entre otras, competencias planificadoras, analíticas, interpretativas, de resolución de problemas y de toma de decisiones, por citar algunas destacadas. Por tanto, los estudiantes a la hora de elaborar el TFM deben considerar que sistematizar un trabajo dentro de su tipología presupone diferentes formas de entenderlo. Para ello, es lícito que conozcan las diferentes opciones en el ámbito de la investigación y las metodologías asociadas a las mismas; es decir; los estudiantes tienen que optar por un tipo de trabajo y organizarlo en torno a un conjunto de elementos que lo delimiten. A esto se suman los intereses y motivaciones de cada persona a la hora de seleccionar un tema o una problemática, definir un contexto de estudio, una fundamentación teórica, una discriminación de sujetos objeto de estudio y una orientación metodológica, ya que todo esto constituye el proceso de investigación, el cual es la llave maestra de su TFM.

En la muestra que hemos analizado -85 TFM- las líneas de investigación elegidas las podemos observar en este gráfico: 


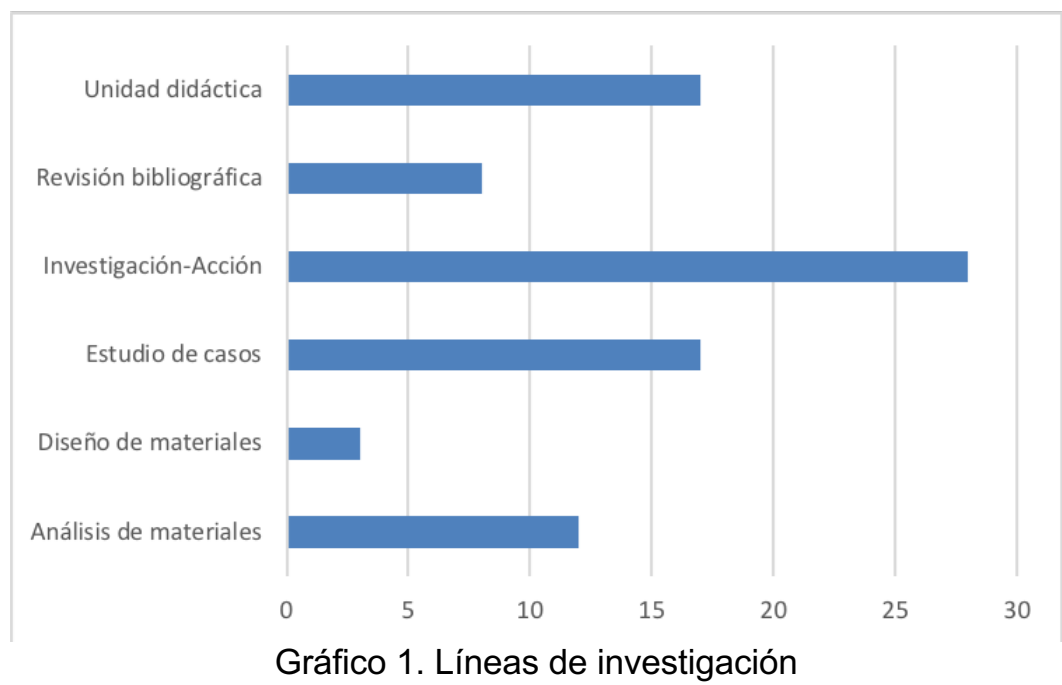

En las producciones estudiadas, las líneas de investigación predominantes en los trabajos fin de máster están orientadas en un alto número hacia la investigación-acción (28 TFM), seguidos del estudio de casos (17) y el diseño de unidades didácticas (17). Decrecen un poco los trabajos sobre análisis de materiales (12) y la revisión bibliográfica (8), sin embargo, el diseño de materiales para el aula de ELE obtiene el número más bajo (3).

Como se puede apreciar, hay un alto predominio de la investigación-acción, un tipo de investigación aplicada cuyo objetivo es solucionar uno o varios problemas de un grupo concreto -en el caso que nos ocupa, el de los aprendientes de español como lengua extranjera-.

Las otras líneas destacadas han sido, por un lado, el estudio de casos prácticos método de investigación muy usado en el ámbito de las ciencias sociales- en los que se estudia el por qué y el cómo de un determinado problema, utilizando diferentes datos, fuentes e instrumentos $y$, por otro, el diseño de unidades didácticas, una herramienta usada frecuentemente por el profesorado para definir el proceso de enseñanzaaprendizaje, considerando el currículum de cada tipo de enseñanza y en el que se delimitan una serie de objetivos, de contenidos, de metodologías didácticas, de herramientas pedagógicas y uno o varios métodos de evaluación. Es obvio que los profesores noveles o aquellos que tienen poca experiencia se decantan por un tipo de trabajos propios de su futuro entorno laboral.

El análisis de materiales es otro tipo de investigación elegida puesto que para los futuros profesores es necesario enfrentarse a la selección, cotejo y análisis de todo tipo de instrumentos para su docencia, dada su heterogeneidad, su adaptabilidad a las metodologías docentes, así como sus diversas formas de implementación en el aula.

Por debajo de estas líneas, en nuestra muestra también se ha optado por los trabajos de revisión bibliográfica -a pesar de que hoy están un tanto denostados en la metodología de la investigación-, ya que los estudiantes utilizan diferentes bases de datos para revisar un determinado tema -siendo muy común los temas de naturaleza gramatical y léxica- y aportar reflexiones y conclusiones sobre la temática estudiada.

El diseño de materiales también se incluye en las líneas de investigación estudiadas. Suelen ser trabajos de creatividad en los que se pretenden hacer innovaciones docentes que cumplan y cubran una serie de objetivos didácticos. Están dirigidos a diversos niveles de lengua, diferentes grupos de aprendizaje -homogéneos y heterogéneos- y todos ellos contribuyen al apoyo docente y a la implementación a través de las TIC.

Por otro lado, Si relacionamos las competencias clave con las líneas de investigación descritas en estos trabajos de investigación, observamos que la competencia a) (organizar situaciones de aprendizaje) obtiene una cifra alta (45 TFM) y se ajusta a las competencias específicas de esta categoría. En general, las producciones de los 
alumnos de posgrado en ELE están orientadas a la reflexión y al uso de la lengua: dicotomía ser/estar; uso y dificultades de los tiempos del pasado; enseñanzaaprendizaje del subjuntivo; aspectos pragmáticos de la comunicación en la lengua extranjera; funcionamiento del artículo determinado e indeterminado, por citar ejemplos recurrentes y destacados.

Asimismo, la planificación de secuencias didácticas es una modalidad habitual en los TFM debido a la necesidad de elaboración de secuencias en las que se planifica el proceso de enseñanza-aprendizaje en torno a una delimitación de objetivos, contenidos, temporalización del proceso de enseñanza, secuenciación de actividades, recursos y materiales didácticos y la delimitación de diferentes formas o procesos de evaluación. Por otro lado, en la investigación también es bastante común dirigir los trabajos hacia el diagnóstico de necesidades de los aprendientes de la lengua extranjera en diferentes contextos de aprendizaje (inmersión, no inmersión, grupos homogéneos y heterogéneos, enseñanza primaria, secundaria, y otras modalidades).

La gestión del aula es otra singularidad común en los trabajos de investigación, ya que estos productos están orientados con frecuencia al aprendizaje significativo, la motivación y la enseñanza afectiva y efectiva.

La competencia c) (implicar a los alumnos en el control de su propio aprendizaje) es menos frecuente (15 TFM) pero también promueve la investigación relacionada con la planificación y el control del proceso de aprendizaje y la determinación de diferentes formas de motivación del alumnado.

Frente a estas dos destacadas competencias el resto del elenco competencial descrito por los profesionales del Instituto Cervantes no se aplica (25 TFM), lo cual es sorprendente ya que de 8 competencias claves en los TFM solo se ha contemplado la categoría a) (Organizar situaciones de aprendizaje), en la que se incide sobre el uso y reflexión de la lengua y la categoría c), en la que predominan los trabajos sobre la gestión de recursos y el control del aprendizaje, como hemos dicho más arriba y como podemos apreciar en el siguiente gráfico:

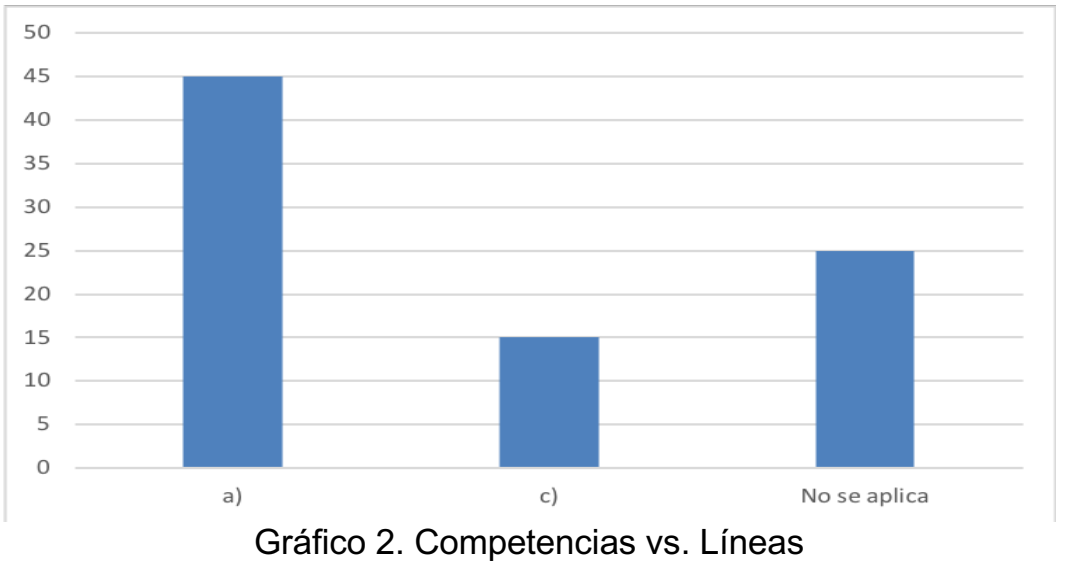

En lo concerniente a las temáticas de los TFM, se observa un claro predominio del componente cultural relacionado con la competencia d) (Facilitar la comunicación intercultural) -16 TFM-. Tal vez se deba a una amplia conciencia de la importancia del desarrollo de la competencia intercultural y a la necesidad de conocimiento del entorno cultural e intracultural de la lengua y a la propia interculturalidad entendida como proceso de conocimiento, diálogo y actuación entre personas de diferentes lenguas y culturas. Así, por ejemplo, proliferan los trabajos sobre los aspectos culturales de la lengua, las variaciones culturales e intraculturales y los aspectos léxicos-culturales implícitos en la comunicación, por citar algunos elementos destacados en la investigación de los futuros profesores de ELE. 
El resto de las temáticas está más repartido y se centra en trabajos de aplicación didáctica sobre las canciones como instrumento en el aula de ELE (6 TFM), sobre la competencia tecnológica (4), la enseñanza de español con fines específicos (turismo) (3), las variedades del español en la enseñanza de ELE (3), entre otros, como se puede apreciar en el siguiente gráfico:

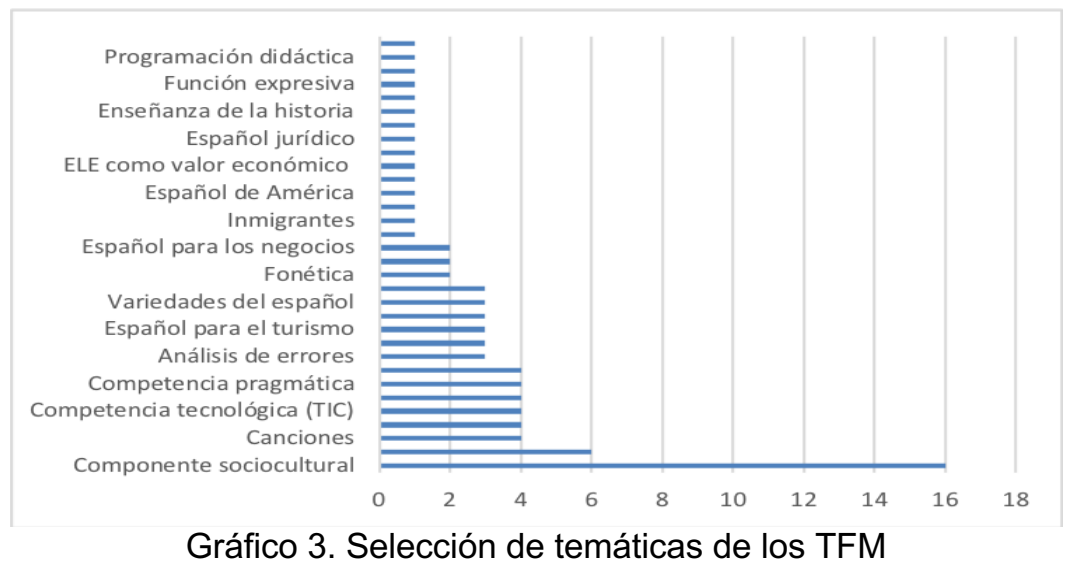

En lo que respecta al cruce de datos sobre las temáticas de los TFM y las competencias claves, se observa la ausencia de trabajos relacionados con las competencias e) (Desarrollarse profesionalmente como profesor de la institución) y g) (Participar activamente en la institución), lo cual es lógico ya que los profesores noveles aún no han tomado conciencia sobre el desempeño profesional dentro de los centros de trabajo e instituciones académicas, por el contrario, los trabajos de investigación se orientan más hacia el dominio de la acción docente y el desarrollo de instrumentos para la docencia ejemplificados, especialmente, en el uso de herramientas tecnológicas, dada su viabilidad didáctica.

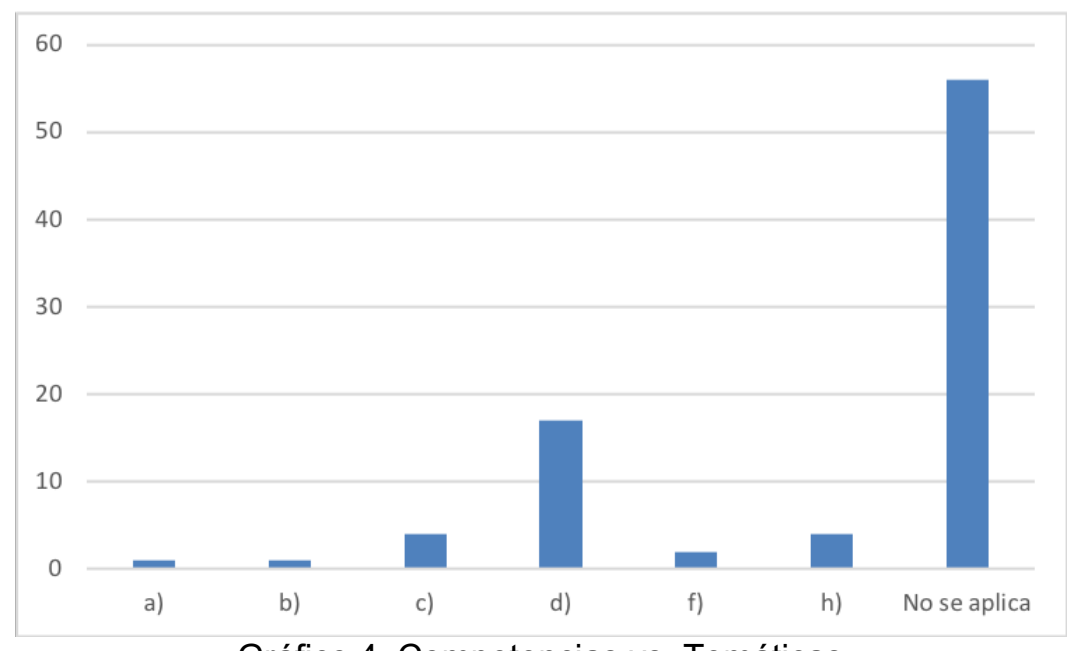

Gráfico 4. Competencias vs. Temáticas

\section{Conclusiones}

A tenor de los casos estudiados, la primera conclusión destacada es que los alumnos egresados de los másteres demandan en sus expectativas, técnicas y recursos para el ejercicio de la docencia en ELE, mientras que los programas de posgrado exigen cierto dominio del proceso de investigación (TFM).

Por otro lado, los futuros profesionales de ELE reclaman primordialmente una mayor formación práctica y un dominio de la acción docente. Aquí cabe no perder de vista que 
las competencias clave del Instituto Cervantes están estudiadas y pensadas para profesionales en activo que acumulan años de experiencia docente, mientras que para los que se inician -profesores noveles- en esta profesión la prioridad se centra en la organización y planificación del proceso de enseñanza-aprendizaje.

En lo que respecta a las tendencias de los alumnos de posgrado en la acción investigadora, las líneas de investigación predominantes son la investigación-acción, el estudio de casos y la planificación de unidades didácticas, aunque en los últimos cursos académicos encontramos TFM dirigidos hacia el estudio e implementación de las TIC en el proceso de enseñanza-aprendizaje.

En cuanto a las temáticas de los TFM, es evidente que la más destacada en las tendencias investigadoras es la sociocultural y todo lo relacionado con la comunicación intercultural. Sin embargo, y aunque existen publicaciones como los de García (1998), se echan en falta trabajos especializados que aborden la formación continua del profesorado y los procesos de investigación, no solo para producir un TFM que les permita a los estudiantes la obtención de un título de posgrado, sino para seguir itinerarios de investigación que les permitan ir creciendo profesionalmente.

Tras este estudio y a modo de valoración final y proyectiva futura, en la formación del profesorado de ELE hemos visto que el alumnado de posgrado tiene una amplia tendencia hacia los trabajos de investigación-acción como método de investigación cualitativa, fundamentado en la acción docente y la mejora de esta; por tanto, el objetivo primordial de los alumnos de posgrado se centra en realizar investigaciones sobre la planificación de acciones y la reflexión sobre aspectos complejos en el entorno docente. Asimismo, y con relación a este tipo de investigación orientado hacia la mejora de la calidad educativa, las temáticas analizadas en los 85 TFM se circunscriben en un alto porcentaje en la cuarta competencia clave -d) - (facilitar la comunicación intercultural), frente al resto de competencias descritas por la institución, las cuales tienen escasa aplicación en el resto de los trabajos de investigación que hemos usado para nuestro estudio.

Para terminar, estas conclusiones tal vez lleven implícito un mensaje de alerta para los formadores del profesorado en general y de ELE u otro tipo de enseñanza en particular: redirigir la investigación hacia los elencos competenciales descritos por las diferentes instituciones académicas, potenciando las diversidades del aula y generar prácticas docentes significativas e innovadoras en las que se aúnen la acción, la reflexión y el diálogo docente como desafío futuro porque, como dijo A. Saint-Exupéry en Le Petit Prince, "será necesario que soporte dos o tres orugas, si quiero conocer las mariposas".

\section{Referencias}

Battaner Arias, M. P. (2002). Investigación en Didáctica de la lengua y la literatura y la consolidación del Área de Conocimiento. Revista de Educación, 328, 59-80.

Cano, E. (2005). Cómo mejorar las competencias docentes. Guía para la autoevaluación y el desarrollo de las competencias del profesorado. Barcelona, España: Graó.

García Llamas, J. L. (1998). La formación permanente del profesorado: motivaciones, realizaciones y necesidades, Educación XX1, $n^{\circ} 1,129-158$. Recuperado de: https://goo.gl/ejNGL9

García, J. L. (1998). La formación permanente del profesorado: motivaciones, realizaciones y necesidades. Educación $X X 1, n^{\circ} 1,129-158$. Recuperado de: https://goo.gl/FGwr3F 
Instituto Cervantes (2012). Las competencias clave del profesorado de lenguas segundas y extranjeras. Recuperado de: https://goo.gl/n9sXKU

Lafuente, C. L., y Egoscozábal, A. M. (2008). Metodologías de la investigación en las ciencias sociales: Fases, fuentes y selección de técnicas. Revista Escuela de Administración de Negocios, (64), 5-18. Recuperado de https://goo.gl/E5bQx9 https://doi.org/10.21158/01208160.n64.2008.450

López-García, Ma . P. (2017). Análisis de modelos competenciales y prácticas docentes innovadoras en la formación del profesorado de ELE y E/L2. En D. G. Níkleva (Ed.), La formación del profesorado de español como lengua extranjera. Necesidades y tendencias (pp. 67-93). Bern: Peter Lang.

López-García, M. ${ }^{a}$ P. y Morales-Cabezas, J. (2015). Necesidades del alumnado en formación como futuros profesionales de español como lengua extranjera. En $\mathrm{N}$. Contreras (ed.) La enseñanza de ELE centrada en el alumno. Actas del XXV Congreso Internacional ASELE, Madrid: ASELE pp. 523-533. Recuperado de: https://goo.gl/GcmbtJ

Martín Peris, E. (2015). Prólogo. En N. Sans y F. Herrera (Dirs.), Formación del profesorado de español. Innovación y reto (pp. 7-8). Barcelona: Difusión.

Mas Torelló, O. (2011). El profesor universitario: sus competencias y formación. Revista de currículum y formación del profesorado, Vol. 14,3, 195-211.

McMullin, K. (2016). Percepción de mejora en el aprendizaje de una segunda lengua. Reidocrea, 5, 22-32.

Níkleva, D. (Ed.) (2017). La formación del profesorado de español como lengua extranjera. Necesidades y tendencias. Bern: Peter Lang. https://doi.org/10.3726/b11686

Níkleva, D. y Rico-Martín, A. M. a (2017). El papel del profesor de español como lengua extranjera o segunda y las necesidades en su formación. En D. G. Níkleva (Ed.), La formación del profesorado de español como lengua extranjera. Necesidades y tendencias (pp. 11-37). Bern: Peter Lang.

Perrenoud, P. (2001). La formación de los docentes en el siglo XXI. Revista de Tecnología Educativa, 3, 503-523

Perrenoud, P. (2004). Diez nuevas competencias para enseñar. Barcelona, España: Graó.

Rodríguez, D. y Valldeoriola, J. (2007). Metodología de la Investigación. Universitat Oberta de Catalunya. Recuperado de https://goo.gl/ATVx2

Saravia Gallardo, M. A. (2011). Calidad del profesorado universitario: un modelo de competencias académicas. Barcelona: Octaedro.

Verdía, E. (2013). Las competencias clave del profesorado y la Parrilla del perfil del profesor de idiomas (EPG): dos documentos de referencia para el desarrollo profesional del docente de lenguas extranjeras. En R. S. Balches (Ed.), Studia Iberystyczne. Nuevas perspectivas de la didáctica en Polonia, tomo XII, pp. 295-314. https://doi.org/10.12797/SI.12.2013.12.16 
Verdía, E. (2016). ¿Por qué y para qué describir las competencias de los profesores de español? En M. ${ }^{a}$ Á. Lamolda González (comp.) y Olga Cruz Moya (ed.), La formación y competencias del profesorado de ELE. XXVI Congreso Internacional de ASELE. Granada: ASELE pp. 37-53. Recuperado de https://goo.gl/iJ97Aq 